\title{
Article \\ Effect of Silica Based Nanoparticles against Plasmodium falciparum and Leishmania infantum parasites
}

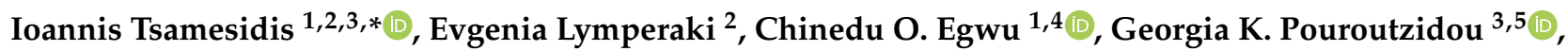 \\ Konstantina Kazeli ${ }^{2}$, Karine Reybier ${ }^{1}$, Sandra Bourgeade-Delmas ${ }^{1}$, Alexis Valentin ${ }^{1}$ and Eleana Kontonasaki ${ }^{3}$ \\ 1 Pharmadev, UMR 152, Université de Toulouse, IRD, UPS, 31000 Toulouse, France; \\ echojay2010@yahoo.com (C.O.E.); karine.reybier-vuattoux@univ-tlse3.fr (K.R.); \\ sandra.bourgeade-delmas@ird.fr (S.B.-D.); alexis.valentin@univ-tlse3.fr (A.V.) \\ 2 Department of Biomedical Sciences, International Hellenic University, 57022 Sindos, Greece; \\ evlimper@gmail.com (E.L.); kazeli.konstantina@gmail.com (K.K.) \\ 3 School of Dentistry, Faculty of Health Sciences, Aristotle University of Thessaloniki, \\ 54124 Thessaloniki, Greece; gpourout@physics.auth.gr (G.K.P.); kont@dent.auth.gr (E.K.) \\ 4 Department of Biochemistry, College of Medicine, Alex-Ekwueme Federal University Ndufu-Alike Ikwo, \\ Abakaliki PMB 1010, Nigeria \\ 5 School of Physics, Faculty of Sciences, Aristotle University of Thessaloniki, 54124 Thessaloniki, Greece \\ * Correspondence: johntsame@gmail.com
}

\section{check for} updates

Citation: Tsamesidis, I.; Lymperaki, E.; Egwu, C.O.; Pouroutzidou, G.K.; Kazeli, K.; Reybier, K.;

Bourgeade-Delmas, S.; Valentin, A.; Kontonasaki, E. Effect of Silica Based Nanoparticles against Plasmodium falciparum and Leishmania infantum parasites. J. Xenobiot. 2021, 11, 155-162. https://doi.org/10.3390/jox11040011

Academic Editors: Yuen-Ki Cheong and Arisbel Cerpa Naranjo

Received: 30 September 2021 Accepted: 5 November 2021 Published: 16 November 2021

Publisher's Note: MDPI stays neutral with regard to jurisdictional claims in published maps and institutional affiliations.

Copyright: (c) 2021 by the authors. Licensee MDPI, Basel, Switzerland. This article is an open access article distributed under the terms and conditions of the Creative Commons Attribution (CC BY) license (https:// creativecommons.org/licenses/by/ $4.0 /)$.

\begin{abstract}
Malaria and Leishmaniasis are two major parasitic diseases, endemic in large areas of tropical countries with high morbidity and mortality across the world. Nanoparticles in small sizes are specifically considered in medicine due to their ability to enter the cells, control the distribution of the administered drug and carry the drug specifically to the place of action. The present study aims to introduce the application of silica nanoparticles as new promising nanotools in malaria and leishmaniasis treatment. Ion doped silica nanomaterials revealed antileishmanial activities indicating the positive role of calcium, magnesium and copper to the surface of the particles against Leishmania parasites. Artemisinin-loaded nanoparticles presented the most promising antiparasitic properties with a sustained release able to overcome the parasite invasion. The sustainable release of artemisinin guarantee both the maintenance of its potential efficacy and also introduce an administration of drug to avoid subsequent drug resistance.
\end{abstract}

Keywords: silica-based nanoparticles; anti-parasitic properties; malaria; leishmanisis; artemisinin

\section{Introduction}

Malaria and leishmaniasis are two important diseases of the tropics caused by Plasmodium and Leishmania. Malaria and leishmaniasis are responsible for at least 400,000 and 20,000 deaths annually, respectively [1-3]. The prevalence of these diseases has stalled and called for renewed strategy in the control approaches. Sometimes, these diseases co-exist as has been reported [4]. Several interventions have been in use; however, these are threatened by the development of resistance to the gold standard drugs for these diseases [3,5]. Resistance to these drugs is multifactorial: short half-lives, high cost that prevents complete treatment or drug overuse. Circumventing these shortcomings can offer hope for the control of these diseases. It is therefore pertinent to design and develop newer malarial and leishmaniasis drugs that can steady the fight against these tropical diseases. Nanotechnology has promises that can overcome some of these challenges [6]. Infusing the active ingredients into carriers that can intermittently release the drugs can overcome the short half-life and ensure full anti-parasitic effect of the drugs [7]. This work is therefore aimed at developing and testing the efficacy and safety of newer antimalarial and anti-leishmaniasis drugs using the nanotechnology. Nanocarriers application in drug delivery is breakeven research and has received a clarion call in biomedicine globally. Moreover addition of magnesium and copper in nanoparticles (NPs) appeared to improve 
the hemocompatibility $[8,9]$ and in the literature it is not still demonstrated the effect of ions to counteract malaria or leishmaniasis. World health organization recommends artemisinin (ART) based combination therapies for the cure of malaria [3], and moreover ART appears in the literature to be active against Leishmania [10]. Artemisinin delivery via nanocarriers could prevent the development of resistance. The aim of our study is firstly to evaluate the antiparasitic properties of undoped and doped NPs and secondly to load nanocarriers with artemisinin and evaluate their synergistic effect with the ions doped to their surfaces in order to reduce the parasite functions.

\section{Materials and Methods}

\subsection{Synthesis of Silica Based Nanoparticles (SbNs)}

Silica-based nanoparticles, $\mathrm{SiO}_{2}(100 \% \mathrm{~mol})(\mathrm{SbN} 1), \mathrm{SiO}_{2} \mathrm{CaOMgO}(55,35$ and $10 \% \mathrm{~mol}$, respectively) ( $\mathrm{SbN} 2), \mathrm{SiO}_{2} \mathrm{CaOMgOCuO}(60,30,7.5$ and $2.5 \%$ mol, respectively) ( $\mathrm{SbN} 3$ ) were successfully synthesized by the Stöber-based sol-gel method. All samples were produced by the hydrolysis of TEOS in a mixture of double-distilled $\mathrm{H}_{2} \mathrm{O}$, ethanol and $\mathrm{HNO}_{3}$, as previously described by our group [11,12]. $\mathrm{Ca}, \mathrm{Mg}$ and $\mathrm{Cu}$ source were added as nitrate salts, while ammonia solution was added drop by drop under stirring and ultrasonic bath. All samples were dried at $75{ }^{\circ} \mathrm{C}$ for 2 days and calcinated in different temperatures (Table 1).

Table 1. Silica-based nanobioceramics (SbNs).

\begin{tabular}{ccc}
\hline \multicolumn{3}{c}{ Silica Based Nanoparticles (SbNs) } \\
\hline Nanoparticles (NPs) & Composition (mol \%) & Calcination Temperature $\left({ }^{\circ} \mathbf{C}\right)$ \\
\hline $\mathrm{SbN} 1$ & $100 \% \mathrm{SiO}_{2}$ & 700 \\
$\mathrm{SbN} 2$ & $55-35-10 \% \mathrm{SiO}_{2} \mathrm{CaOMgO}$ & 1000 \\
$\mathrm{SbN} 3$ & $60,30,7.5$ and $2.5 \%$ & 700 \\
$\mathrm{mSbN}$ & $\mathrm{SiO}_{2}-\mathrm{CaO}-\mathrm{MgO}^{-\mathrm{CuO}}$ & 600 \\
\hline
\end{tabular}

The synthesis of silica-based mesoporous nanocarriers $\left(100 \% \mathrm{~mol} \mathrm{SiO}_{2}\right)(\mathrm{mSbN})$, was performed through a modified sol-gel method, as described before [13]. The cetyltrimethylammonium bromide (CTAB) was used as the agent for the mesoporous structure. CTAB was added in a solution of sodium hydroxide ( $\mathrm{NaOH}$, alkaline medium), and stirred for $30 \mathrm{~min}$. Then tetraethyl orthosilicate (TEOS) was added dropwise and stirred vigorously for $2 \mathrm{~h}$. The synthesized sample was dried at $60{ }^{\circ} \mathrm{C}$ overnight and calcinated at $600{ }^{\circ} \mathrm{C}$ for $5 \mathrm{~h}$ to remove CTAB.

\subsection{Fourier Transform Infrared Spectroscopy (FTIR)}

Perkin Elmer Spectrometer Spectrum 1000 was used for the Fourier Transform Infrared Spectroscopy (FTIR) analysis. The spectra of the synthesized samples were collected in the transmittance mode in the range of $400-4000 \mathrm{~cm}^{-1}$. The pellets of all samples with $\mathrm{KBr}$ powder (ratio of 1:100) were prepared under pressure of 7 tons.

\subsection{ART-Loaded NP (ART-mSbN) and UHPLC/HRMS Analysis of ART Concentration}

Mesoporous NPs in alkaline solution was used for the loading of $1 \mathrm{mM}$ artemisinin after stirring ( $300 \mathrm{rpm}$ ) at room temperature for 1 day. The ART-mSbN were separated from the suspension by centrifugation at $5000 \times g$ for $15 \mathrm{~min}$ and was analyzed onto a UHPLC Kinetex EVO C18 $1.7 \mu \mathrm{m}, 2.1 \times 100$ mm column (Phenomenex, France) using UHPLC/HRMS system as previously described [14]. The following equation was used to calculate the loading capacity (LC) of artemisinin in NPs.

$$
\text { Loading capacity }=\frac{\text { total amount of drug } \times \text { free amount of drug }}{\text { weight of nanoparticules }}
$$


For the drug release studies, supernatants at different time points $(0,6,17,21,24,41$, $48,72,96 \mathrm{~h}$ ) were collected and stored into the deep freeze. The artemisinin amount was quantified as previously described. The samples of each experiment were analyzed in triplicate.

\subsection{Blood Sample Collection}

Whole blood from healthy donors was collected after obtaining the informed consent to participate in the study. The separation of the erythrocytes from plasma and leukocytes was performed by washing them three times with Phosphate buffer saline (PBS). The study was conducted in accordance with Good Clinical Practice guidelines and the Declaration of Helsinki and was approved by Etablissement Français du Sang (EFS, Toulouse, France), responsible for ethic statements.

\subsection{Antileishmanial Activity on L. infantum Axenic Amastigotes}

L. infantum promastigotes (MHOM/MA/67/ITMAP-263, CNR Leishmania, Montpellier, France, expressing luciferase activity) were cultivated in RPMI 1640 medium supplemented with $10 \%$ fetal calf serum (FCS), $2 \mathrm{mM}$ L-glutamine and antibiotics (100 U/mL penicillin and $100 \mu \mathrm{g} / \mathrm{mL}$ streptomycin) and harvested in logarithmic phase of growth by centrifugation at $900 \times g$ for $10 \mathrm{~min}$. The acidified promastigotes were incubated for $24 \mathrm{~h}$ at $37^{\circ} \mathrm{C}$ in a ventilated flask to transform promastigotes into amastigotes. The effects of the tested nanoparticles on the growth of L. infantum amastigotes were assessed as follows:

L. infantum amastigotes were incubated at a density of $2 \times 10^{6}$ parasites $/ \mathrm{mL}$ in sterile 96-well plates with various concentrations of compounds dissolved in PBS (final concentration less than $0.5 \% v / v$ ), in duplicate. Appropriate controls, PBS and amphotericin, were added to each set of experiments. After a $48 \mathrm{~h}$ incubation period at $37^{\circ} \mathrm{C}$, each plate-well was then microscopically-examined to detect any precipitate formation. To estimate the luciferase activity of axenic amastigotes, $80 \mu \mathrm{L}$ of each well were transferred to white 96-well plates, Steady Glow ${ }^{\circledR}$ reagent (Promega, London, UK) was added according to manufacturer's instructions, and plates were incubated for $2 \mathrm{~min}$. The luminescence was measured in Microbeta Luminescence Counter (PerkinElmer, Rome, Italy).

\subsection{Antiplasmodial Activity}

For the in vitro testing of the efficacy of the nanoparticles, the Plasmodium falciparum FCB1 strain was used. The parasites were kept in RPMI 1640 at 5\% serum at 2\% hematocrit at $37^{\circ} \mathrm{C} 5 \% \mathrm{CO}_{2}$ atmosphere. For each experiment, the parasites were tightly synchronized by $5 \%$ D-sorbitol treatment at the ring stage (0-24 h) (Lambros and Vanderberg, 1979). The inhibitory concentration $\left(\mathrm{IC}_{50}\right.$ ) of the molecules were determined by light microscopy method as the gold standard method by WHO $[15,16]$. Tightly synchronized rings at $1 \%$ parasitemia were treated with different concentrations of each nanoparticle preparation, using artemisinin as control molecule and incubated for $48 \mathrm{~h}$. The molecules were then washed off three times in PBS.

\subsection{Assessment of Parasitemia by Light Microscopy}

The evaluation of the parasitemia and the parasite stage was performed with thin smears and prepared at specific time points ( $24 \mathrm{~h}$ of incubation) and stained with DiffQuick stain. Three observers examined a minimum of 10,000 cells microscopically. The experiments were performed at least in triplicate.

\section{8. $I C_{50}$ Measurement}

Antiplasmodial activity: The program, ICEstimator software version 1.2, was used to estimate the half maximal inhibitory concentrations of the different nanoparticles estimates using a nonlinear regression function of the $\mathrm{R}$ software version 1.2.

Antileishmanial activity: Efficient concentration 50\% (EC50) was defined as the concentration of drug required to inhibit by $50 \%$ the metabolic activity of L. infantum amastigotes 
compared to control. EC50 values were calculated by non-linear regression analysis processed on dose response curves, using TableCurve 2D V5 software. EC50 values represent the mean of three independent experiments.

\section{Results and Discussion}

\subsection{Physicochemical Characteristics of SbNs}

The synthesized nanoparticles were of round shape with sizes of $2.05 \mathrm{~nm} \pm 0.39$ for $\mathrm{SbN} 1,6.82 \pm 1.27$ for SbN2 and $5.70 \pm 1.06$ for SbN3 and $408.80 \pm 0.05$ for $\mathrm{mSbN}[8,9]$. All samples presented negative $\zeta$-potential with the highest value recorded for the sample $\mathrm{mSbN}[8,9]$. The FTIR spectra of the synthesized nanoparticles revealed the characteristic bands of silicate glasses in all spectra, assigned to the broad peak around $900-1200 \mathrm{~cm}^{-1}$ attributed to the symmetric stretching vibration of $\mathrm{Si}-\mathrm{O}-\mathrm{Si}$ and the peak around $470 \mathrm{~cm}^{-1}$ attributed to the bending vibration of the $\mathrm{Si}-\mathrm{O}-\mathrm{Si}$ bonds (Figure 1) [11-13]. Silica nanoparticles $(\mathrm{SbN} 1)$ and mesoporous nanoparticles $(\mathrm{mSbN})$ presented an additional peak around $814 \mathrm{~cm}^{-1}$ attributed to the symmetric stretching of the $\mathrm{Si}-\mathrm{O}-\mathrm{Si}$ bonds. Moreover, the spectra of $\mathrm{SbN} 2$ and $\mathrm{SbN} 3$ presented a shoulder around $902 \mathrm{~cm}^{-1}$ related to the vibration of the $\mathrm{Si}-\mathrm{O}-\mathrm{Ca}$ bonds [13].

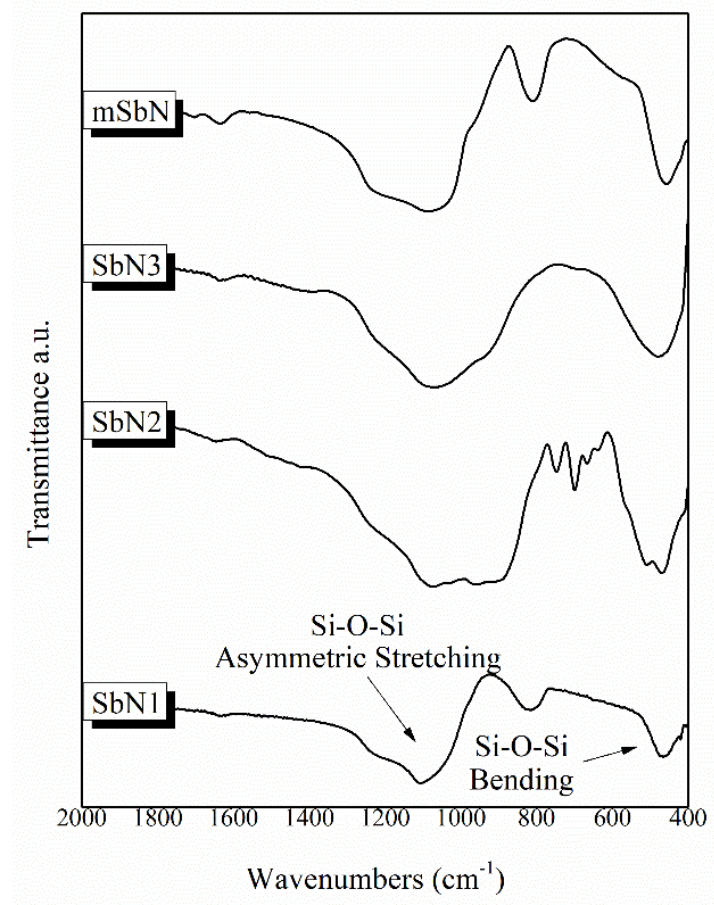

Figure 1. FTIR spectra of the synthesized samples.

\subsection{Anti-Leishmania Activity of Nanoparticles}

The anti-leishmanial activity of the tested nanoparticles are presented in Table 2 using amphotericin $\mathrm{B}$ as positive control. The $\mathrm{IC}_{50 \text { s }}$ were generally low in comparison to the gold standard, amphotericin B. The most effective was ART-mSbN, with an $\mathrm{IC}_{50}$ of $1.43 \mu \mathrm{g} / \mathrm{mL}$ which was lower than unloaded $m S b N(10 \mu \mathrm{g} / \mathrm{mL})$ (Figure 2). The anti-leishmanial effect of artemisinin has already been demonstrated [10], but was firstly presented via nanocarriers to counteract the parasites. This shows the nanotechnology absorbed the activity of ART to reduce the L. infantum promastigotes activity. However, a reported resistance to some antileishmanial drugs including artemisinin $[17,18]$ calls for a paradigm shift in the administration and choice of drugs. An improved delivery through nanotechology could be fully exploited in the development of newer agents in the treatment of Leishmania. From all the nanoparticles, the $\mathrm{SbN} 3$ presented $\mathrm{IC}_{50}$ values close to the values of ART$\mathrm{mSbN}$. This particular nanoparticle was copper-doped. This activity maybe correlated to copper ions, as copper has been used as a chemotherapeutic agent against various parasitic 
infections [19]. In the study of Singh et al., a copper compound (copper salisylaldoximeCuSAL) presented potent anti-leishmanial activity [20], while in a recent study, growth media supplemented with copper resulted in a gradual decline in Leishmania parasites survivability with increasing copper concentration [21]. More research is needed on investigating possible routes for utilizing copper or copper-doped nanoparticles towards chemotherapy of human leishmaniasis.

Table 2. Effects of nanoparticles against Leishmania amastigotes in vitro.

\begin{tabular}{cc}
\hline Nanoparticles/Drug & IC $_{\mathbf{5 0}}(\boldsymbol{\mu g} / \mathbf{m L})$ \\
\hline Amphotericin B & $6.5 \times 10^{-2}$ \\
SbN1 & $>10 \pm 1.1$ \\
SbN2 & $>10 \pm 1.52$ \\
SbN3 & $2.46 \pm 0.35$ \\
mSbN & $>10 \pm 0.91$ \\
ART-mSbN & $1.43 \pm 0.25$ \\
\hline
\end{tabular}

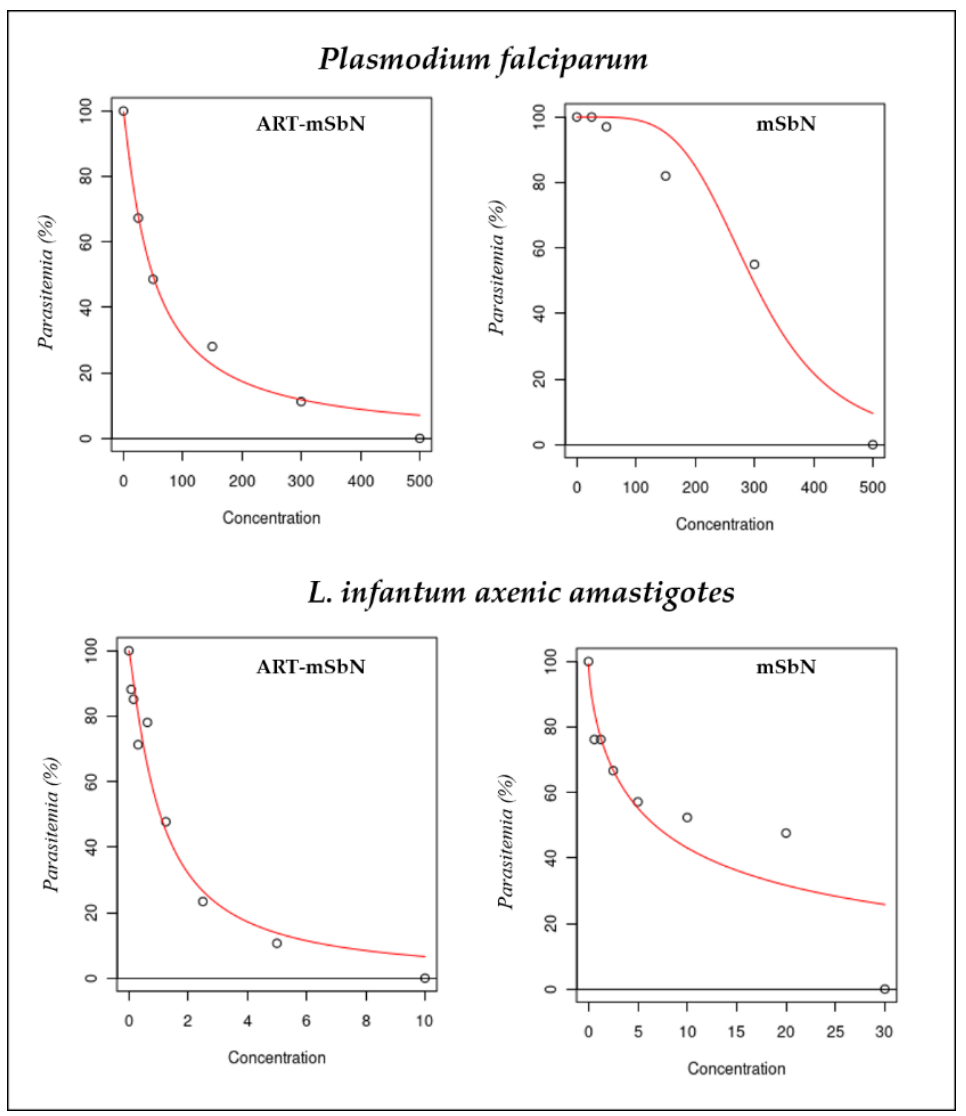

Figure 2. Rapresentation of the half maximal inhibitory concentrations (IC50s) non-linear regression curve of ART-mSbN and $\mathrm{mSbN}$ for Plasmodium falciparum and L. infantum axenic amastigotes.

\subsection{Antimalarial Activity of Nanoparticles}

The antimalarial activity of the tested nanoparticles was evaluated after $24 \mathrm{~h}$ of incubation and is presented in Table 3 using artemisinin as positive control. As expected, of all the nanoparticles tested, $A R T-m S b N$ was the most effective $\left(\mathrm{IC}_{50}=50 \mu \mathrm{g} / \mathrm{mL}\right.$ ) while the least effective was $\mathrm{SbN1}$. The $\mathrm{IC}_{50}$ of the NPs are generally less than $500 \mu \mathrm{g} / \mathrm{mL}$. The highest efficacy recorded from $A R T-m S b N$ could be indicative of a retention of the artemisinin activity that was embedded in the mesoporous structure of these nanoparticles (Figure 2). The morphology of parasites was significantly affected and is presented in Figure 3. ART-mSbN treated parasites showed detectable effects to their maturation and 
ability of invasion. The release capacity of the $A R T-m S b N$ was recorded and presented a sustained release until the first $48 \mathrm{~h}$ (Table 4). A slow-going release at the first $6 \mathrm{~h}$ started followed by a continuous release, eventually reaching a $69 \%$ as artemisinin release capacity.

Table 3. Antimalarial effect of nanoparticles.

\begin{tabular}{cc}
\hline Nanoparticles/Drug & IC $_{\mathbf{5 0}}(\boldsymbol{\mu g} / \mathbf{m L})$ \\
\hline Artemisinin & $9.8 \mathrm{nM}=2.8 \times 10^{-3}$ \\
SbN1 & $325 \pm 35$ \\
SbN2 & $200 \pm 28$ \\
SbN3 & $225 \pm 15$ \\
mSbN & $300 \pm 29$ \\
ART-mSbN & $50 \pm 15$ \\
\hline
\end{tabular}

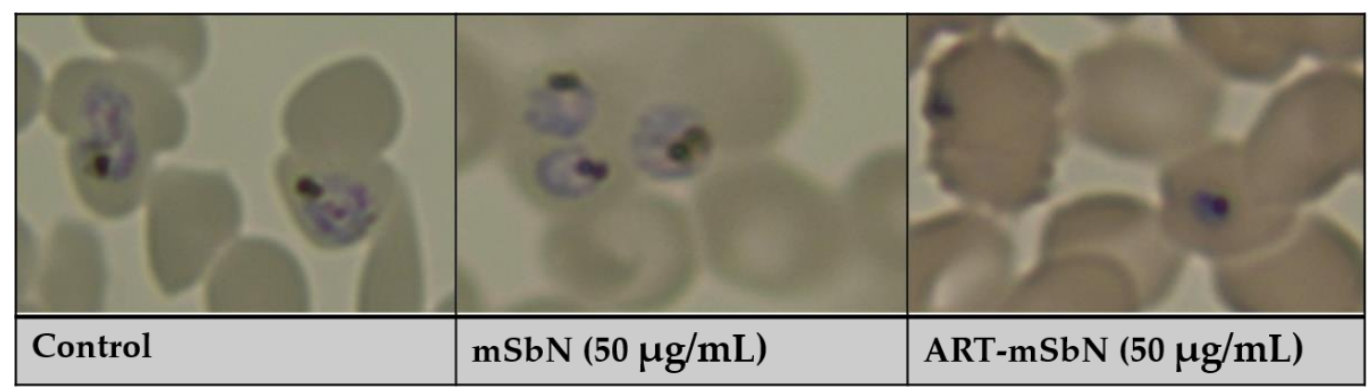

Figure 3. Morphological changes in P. falciparum after treatment with $\mathrm{mSbN}$ and ART-mSbN.

Table 4. Artemisinin release capacity (\%) of ART-mSbN in a time range of up to $96 \mathrm{~h}$.

\begin{tabular}{cc}
\hline Time (hours) & Artemisinin Release Capacity (\%) of ART-mSbN \\
\hline 6 & 25 \\
17 & 40 \\
24 & 42 \\
41 & 54 \\
48 & 62 \\
72 & 67 \\
96 & 69 \\
\hline
\end{tabular}

Artemisinin and its derivatives remain the gold standard in the treatment of malaria [3]. However its short halflife challenges its future usefulness in malaria control, as Plasmodium easily develop resistance due to short period of exposure. A technology that can intermittently release the molecule, overcoming the shortlife shortfall will be useful in the fight against resistance development to artemisinin. Nanotechnology has been reputed for this intermitent releases and targetted drug delivery of molecules [22], ensuring a sustained activity of different substances. These preliminary results suggest that by tailoring the properties of mesoporous nanoparticles, a prolonged and sustained release can be achieved, promoting the beneficial action of artemisinin. Future research should focus on mechanisms for increased grafting of artemesinin into and onto the surface of mesoporous nanoparticles for efficient drug loading and release. Taking into consideration, the involvment of intraerythrocytic superoxide and hydrogen peroxide generation to activate artemisinin [23], the administration of artemisinin could be better enhanced via this system.

It is important to note that the above pharmacological activities of the tested nanoparticles was achieved without them having hemolytic effects as previously presented by Tsamesidis et al. [8,9]. The same set of hemocompatibility experiments was performed to confirm the biocompatibility of NPs in parastizied erythrocytes too (Supplemental Figure S1). All the tested biomaterials presented a biocompatible behaviour (hemolysis $<2 \%$ ) which is considered as non-hemolytic according to ASTM F 756-08 (ASTMF-756, 2009). This underscores the safety of these silica-based nanoparticles as potential agents in the tretament of 
lesihmania and malaria, although further research is needed on whole blood components, such as platelets and white blood cells.

Supplementary Materials: The following are available online at https:/ / www.mdpi.com/article/10 .3390/jox11040011/s1, Figure S1: Hemolytic effect of NPs in parasitized erythrocytes.

Author Contributions: Conceptualization: E.K. and I.T.; methodology: I.T., E.K., G.K.P., E.L., C.O.E., K.K., K.R., S.B.-D. and A.V.; formal analysis: I.T., E.K., E.L., G.K.P., C.O.E. and S.B.-D.; investigation: I.T., E.K. and S.B.-D.; resources: K.R., E.K., S.B.-D. and A.V.; writing-original draft preparation: I.T., E.K. and C.O.E.; writing-review and editing: E.K., I.T., E.L. and K.R.; supervision: I.T and E.K. All authors have read and agreed to the published version of the manuscript.

Funding: This research received no external funding.

Institutional Review Board Statement: The study was conducted in accordance with Good Clinical Practice guidelines and the Declaration of Helsinki and was approved by Etablissement Français du Sang (EFS, Toulouse, France), responsible for ethic statements.

Informed Consent Statement: Informed consent was obtained from all subjects involved in the study.

Data Availability Statement: Data are contained within the article.

Conflicts of Interest: The authors declare no conflict of interest.

\section{References}

1. WHO. Leishmaniasis. Available online: https://www.who.int/health-topics/leishmaniasis\#tab=tab_1 (accessed on 4 November 2021).

2. PAHO/WHO Fact Sheet: Neglected Infectious Diseases_Leishmaniasis I Enhanced Reader. Available online: https: / /www.paho. org/en/topics/leishmaniasis (accessed on 4 November 2021).

3. WHO. World Malaria Report 2020: 20 Years of Global Progress and Challenges. Available online: https://www.who.int/ publications/i/item/9789240015791 (accessed on 4 November 2021).

4. Aschale, Y.; Ayehu, A.; Worku, L.; Tesfa, H.; Birhanie, M.; Lemma, W. Malaria-visceral leishmaniasis co-infection and associated factors among migrant laborers in West Armachiho district, North West Ethiopia: Community based cross-sectional study. BMC Infect. Dis. 2019, 19, 239. [CrossRef] [PubMed]

5. Chakravarty, J.; Sundar, S. Drug resistance in leishmaniasis. J. Glob. Infect. Dis. 2010, 2, 167. [CrossRef] [PubMed]

6. Feng, J.; Thian, E.S. Applications of nanobioceramics to healthcare technology. Nanotechnol. Rev. 2013, 2, 679-697. [CrossRef]

7. Avitabile, E.; Senes, N.; D'Avino, C.; Tsamesidis, I.; Pinna, A.; Medici, S.; Pantaleo, A. The potential antimalarial efficacy of hemocompatible silver nanoparticles from Artemisia species against P. falciparum parasite. PLoS ONE 2020, 15, e0238532. [CrossRef] [PubMed]

8. Tsamesidis, I.; Pouroutzidou, G.K.; Lymperaki, E.; Kazeli, K.; Lioutas, C.B.; Christodoulou, E.; Perio, P.; Reybier, K.; Pantaleo, A.; Kontonasaki, E. Effect of ion doping in silica-based nanoparticles on the hemolytic and oxidative activity in contact with human erythrocytes. Chem. Biol. Interact. 2020, 318, 108974. [CrossRef] [PubMed]

9. Tsamesidis, I.; Kazeli, K.; Lymperaki, E.; Pouroutzidou, G.K.; Oikonomou, I.M.; Komninou, P.; Zachariadis, G.; Reybier, K.; Pantaleo, A.; Kontonasaki, E. Effect of Sintering Temperature of Bioactive Glass Nanoceramics on the Hemolytic Activity and Oxidative Stress Biomarkers in Erythrocytes. Cell. Mol. Bioeng. 2020. [CrossRef] [PubMed]

10. Sen, R.; Ganguly, S.; Saha, P.; Chatterjee, M. Efficacy of artemisinin in experimental visceral leishmaniasis. Int. J. Antimicrob. Agents 2010, 36, 43-49. [CrossRef] [PubMed]

11. Pouroutzidou, G.K.; Theodorou, G.S.; Kontonasaki, E.; Tsamesidis, I.; Pantaleo, A.; Patsiaoura, D.; Papadopoulou, L.; Rhoades, J.; Likotrafiti, E.; Lioutas, C.B.; et al. Effect of ethanol/TEOS ratios and amount of ammonia on the properties of copper-doped calcium silicate nanoceramics. J. Mater. Sci. Mater. Med. 2019, 30. [CrossRef] [PubMed]

12. Pouroutzidou, G.K.; Theodorou, G.S.; Kontonasaki, E.; Papadopoulou, L.; Kantiranis, N.; Patsiaoura, D.; Chrissafis, K.; Lioutas, C.B.; Paraskevopoulos, K.M. Synthesis of a bioactive nanomaterial in the ternary system SiO2-CaO-MgO doped with CuO: The effect of Ball milling on the particle size, morphology and bioactive behavior. AIP Conf. Proc. 2019, 200005. [CrossRef]

13. Pouroutzidou, G.K.; Liverani, L.; Theocharidou, A.; Tsamesidis, I.; Lazaridou, M.; Christodoulou, E.; Beketova, A.; Pappa, C.; Triantafyllidis, K.S.; Anastasiou, A.D.; et al. Article synthesis and characterization of mesoporous mg-and sr-doped nanoparticles for moxifloxacin drug delivery in promising tissue engineering applications. Int. J. Mol. Sci. 2021, 22, 577. [CrossRef] [PubMed]

14. Tsamesidis, I.; Gkiliopoulos, D.; Pouroutzidou, G.K.; Lymperaki, E.; Papoulia, C.; Reybier, K.; Perio, P.; Paraskevopoulos, K.M.; Kontonasaki, E.; Theocharidou, A. Effect of Artemisinin-Loaded Mesoporous Cerium-Doped Calcium Silicate Nanopowder on Cell Proliferation of Human Periodontal Ligament Fibroblasts. Nanomaterials 2021, 11, 2189. [CrossRef] [PubMed]

15. WHO. World Malaria Report 2017; WHO: Geneva, Switzerland, 2017. 
16. Pau, M.C.; Pantaleo, A.; Tsamesidis, I.; Hoang, H.; Tran, A.T.; Nguyen, T.L.H.; Phan, T.H.G.; Nu, P.A.T.; Ngo, T.M.C.; Marchetti, G.; et al. Clinical impact of the two ART resistance markers, K13 gene mutations and DPC3 in Vietnam. PLoS ONE 2019, 14, e0214667. [CrossRef] [PubMed]

17. Gruessner, B.M.; Cornet-Vernet, L.; Desrosiers, M.R.; Lutgen, P.; Towler, M.J.; Weathers, P.J. It is not just artemisinin: Artemisia sp. for treating diseases including malaria and schistosomiasis. Phytochem. Rev. 2019, 18, 1509-1527. [CrossRef] [PubMed]

18. Verma, A.; Ghosh, S.; Salotra, P.; Singh, R. Artemisinin-resistant Leishmania parasite modulates host cell defense mechanism and exhibits altered expression of unfolded protein response genes. Parasitol. Res. 2019, 118, 2705-2713. [CrossRef] [PubMed]

19. Norouzi, R. A review on most nanoparticles applied against parasitic infections. J. Biol. Today's World 2017, 6, 196-203. [CrossRef]

20. Singh, M.K.; Bhaumik, S.K.; Karmakar, S.; Paul, J.; Sawoo, S.; Majumder, H.K.; Roy, A. Copper salisylaldoxime (CuSAL) imparts protective efficacy against visceral leishmaniasis by targeting Leishmania donovani topoisomerase IB. Exp. Parasitol. 2017, 175, 8-20. [CrossRef] [PubMed]

21. Paul, R.; Banerjee, S.; Sen, S.; Dubey, P.; Bachhawat, A.K.; Datta, R.; Gupta, A. The novel leishmanial Copper P-type ATPase plays a vital role in intracellular parasite survival. bioRxiv 2021. [CrossRef]

22. Cattaneo, A.G.; Gornati, R.; Sabbioni, E.; Chiriva-Internati, M.; Cobos, E.; Jenkins, M.R.; Bernardini, G. Nanotechnology and human health: Risks and benefits. J. Appl. Toxicol. 2010, 30, 730-744. [CrossRef] [PubMed]

23. Tsamesidis, I.; Pério, P.; Pantaleo, A.; Reybier, K. Oxidation of erythrocytes enhance the production of reactive species in the presence of artemisinins. Int. J. Mol. Sci. 2020, 21, 4799. [CrossRef] [PubMed] 